\section{Conflictos éticos en las autorías de trabajos científicos}

\author{
ASTRID VON OETINGER ${ }^{1, \mathrm{a},}$ KABIR P. SADARANGANI ${ }^{2,3, \mathrm{a}}$, \\ SOFÍA P. SALAS ${ }^{4}$
}

\section{Ethical conflicts in the authorship of scientific papers}

The frequency of conflicts about authorship of publications has increased along with the increase in the number of people involved in scientific work. Some of the factors that strongly influence the generation of conflicts and malpractices in authorship definition of scientific publications are the pressure of academia, economic incentives from the pharmaceutical industry in the field of biomedicine and authors' wishes and expectations of recognition, among other factors. The article analyzes this problem, increasingly common in the field of medicine and related areas. Special attention is devoted to the prevailing laws in our country and international guidelines related to intellectual property and authorship of scientific publications, respectively. However, the ethical commitment, intellectual honesty and truthfulness of each of the authors about what is reported seems to be the decisive factor for the solution to these authorship conflicts.

(Rev Med Chile 2016; 144: 1473-1478)

Key words: Authorship; Ethics in Publishing; Fraud, Scientific; Scientific Misconduct.

\author{
'Escuela de Kinesiología, Facultad \\ de Ciencias de la Rehabilitación, \\ Universidad Andres Bello. \\ 2Escuela de Kinesiología, \\ Facultad de Ciencias de la Salud, \\ Universidad San Sebastián. \\ 32Departamento de Educación \\ Física, Deporte y Motricidad \\ Humana, Facultad de Formación \\ del Profesorado y Educación, \\ Universidad Autónoma de \\ Madrid, España. \\ ${ }^{4}$ Facultad de Medicina, \\ Universidad Diego Portales. \\ aKinesiólogos. \\ Conflictos de interés: Ninguno \\ que declarar. Este trabajo no \\ obtuvo financiamiento. \\ Recibido el 1 de abril de 2016, \\ aceptado el 6 de septiembre de \\ 2016. \\ Correspondencia a: \\ Astrid von Oetinger Giacoman \\ Fernández Concha 700, Las \\ Condes \\ Teléfono: 56226618402 \\ astridvon@gmal.com/astrid. \\ vonoetinger@unab.cl
}

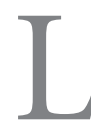

a publicación de un trabajo científico finaliza un largo proceso y constituye una responsabilidad ética para todos los investigadores, siendo un aspecto relevante quiénes califican como autor, puesto que existe una relación entre los hechos de la naturaleza que se describen en un determinado artículo, los autores que los describen y la propiedad intelectual de estos autores respecto de sus escritos ${ }^{1,2}$. Desde una perspectiva histórica, las fuerzas del mercado y los derechos de autor fueron factores importantes en la conformación del papel del autor de un trabajo científico, aunque este camino no estuvo exento de dificultades ${ }^{2}$.

El uso correcto de las autorías establece responsabilidad y el debido crédito por la información científica comunicada en las publicaciones ${ }^{3}$. A nivel internacional, existen varios Comités Éticos para editores que regulan estos aspectos; el más conocido es el "Comité Internacional de Editores de Revistas Médicas” (ICMJE), creado en el año 1979 en Canadá por un grupo de editores con el objeto de revisar los estándares éticos en las publicaciones biomédicas ${ }^{4}$. Recientemente se actualizaron estas recomendaciones, las que son exigidas por revistas internacionales y chilenas ${ }^{5-8}$.

En Chile, la Ley No 17.336 regula la propiedad intelectual, protegiendo "los derechos que, por el solo hecho de la creación de la obra, adquieren los autores de obras de la inteligencia en los dominios literarios, artísticos y científicos... y los derechos conexos que ella determina. El derecho de autor comprende los derechos patrimonial y moral, que protegen el aprovechamiento, la paternidad y la integridad de la obra" ". No obstante, la autoría científica no obedece a las leyes del copyright, sino que corresponde a una demanda más bien simbólica, como sería el reconocimiento que otorga la comunidad de pares ${ }^{2}$.

A pesar de que existen criterios obligatorios respecto de las autorías científicas y que incluso 
la Revista Médica de Chile exige completar un formulario de Declaración de Autoría ${ }^{6}$, este tema sigue generando conflictos. En este trabajo, junto con actualizar los criterios vigentes de autoría científica, analizaremos los principales problemas relacionados con las autorías, incluyendo algunos aspectos éticos ${ }^{10}$. Debe hacerse presente que el tema de las autorías es uno sólo de los conflictos relacionados con mala conducta científica, pero por motivos de espacio, sólo nos abocaremos a éste.

\section{¿Quiénes deben ser autores de una publicación científica?}

El ICMJE recomienda que la autoría se base en cuatro criterios, los que deben ser cumplidos por todos los co-autores (Tabla 1$)^{4}$. Además de ser responsable de las partes del trabajo en las cuales participó, el autor principal debe identificar qué co-autores participaron en las distintas secciones del artículo y debe confiar en la integridad de las contribuciones de aquellos. Los autores que no cumplan con todos los criterios señalados pueden ser reconocidos en el apartado de agradecimientos ${ }^{4}$. Ejemplos de actividades que no cumplen con los criterios de autoría son la mera adquisición de fondos; supervisión general del grupo de investigación; apoyo administrativo; asistencia en la redacción del trabajo; edición técnica; traducción al idioma requerido; asesoría científica; ejecución del análisis estadístico; procesamiento de muestras; o la atención a pacientes del estudio ${ }^{11}$.

Estos criterios de autoría pretenden reservar la categoría de autor sólo a los que merecen el crédito correspondiente y asumen la responsabilidad pública del artículo científico, pero no pretenden ser un medio de exclusión de pares. Así, todo investigador que cumpla el primer criterio debe tener la oportunidad de participar en la revisión, elaboración y aprobación final del manuscrito, cumpliendo con los criterios siguientes; la mayoría de los editores exigen al autor principal obtener la autorización por escrito de los otros autores ${ }^{4}$. La determinación de quién califica o no para autoría, o el arbitraje de conflictos de autoría, son de responsabilidad colectiva de los autores y no del comité de editores ${ }^{12}$. Habiendo desacuerdo, sugerimos que sea la institución donde se realizó la investigación quien tome la decisión final, para lo cual debiesen contar con instancias preestablecidas para resolver estos conflictos ${ }^{4,12}$. La solicitud de eliminación o adición de un autor después de presentado el manuscrito, da la facultad al comité de editores de exigir una explicación y firma de la declaración de acuerdo para el cambio por todos los autores, incluyendo el que fue retirado o añadido ${ }^{4,13}$.

\section{Autor para la correspondencia y orden de los autores}

El autor para la correspondencia es quien asume la responsabilidad primaria para la comunicación con la revista durante la presentación de manuscritos, revisión por pares y el proceso de publicación. Asegura además que todos los requisitos administrativos de la revista se han completado correctamente, aunque estas funciones pueden delegarse en otros coautores ${ }^{4,14}$. Este autor debe estar disponible después de la publicación para responder a las críticas de trabajo y cooperar con cualquier petición de la revista respecto de información adicional. Aunque el autor principal es responsable de la correspondencia con la revista, el ICMJE recomienda que los editores envíen copias

\section{Tabla 1. Criterios de autoría de trabajos científicos}

1. Aportar contribuciones sustanciales a la concepción o diseño del estudio; o a la adquisición, análisis o interpretación de los datos para el trabajo.

2. Participar en la redacción del trabajo o en su revisión crítica, contribuyendo a su contenido intelectual.

3. Aprobar de la versión final a ser publicada.

4. Asumir la responsabilidad pública de todos los aspectos del estudio, asegurando que contestará de manera adecuada cualquier pregunta relacionada con el estudio.

Adaptado de referencia 4 
de la correspondencia a todos los autores que figuran en ésta ${ }^{4,14}$. En general, el autor más relevante es quien va en primer lugar, el cual se supone ha realizado la mayor contribución al trabajo, especialmente en el laboratorio o en terreno; el que va último, suele ser el autor de mayor experiencia, contribuyendo con su guía y apoyo intelectual ${ }^{13,15}$. Dado que existen diferencias culturales al respecto, proponemos que estos temas sean acordados previo al estudio.

\section{Multiautorías en trabajos científicos}

En las últimas décadas ha existido un aumento sustantivo en el número de artículos de multiautoría ${ }^{16,17}$. Este tipo de situaciones ha cuestionado la contribución de cada autor y la validez de su autoría $^{14}$.Las posibles explicaciones al fenómeno de las multiautorías son muchas. Cabe destacar el aumento en las investigaciones multicéntricas, en donde el número de autores aumenta debido a la mayor colaboración necesaria para realizar la misma. Sin embargo, otras causas serían la realización indiscriminada de artículos de revisión, la importancia de publicar para la promoción académica y obtener oportunidades de financiamiento, el uso inadecuado de la situación de poder por algunos académicos, y la inexperiencia de los propios autores, entre otros factores ${ }^{4,12}$. Más aún, la presión en lo académico para publicar en revistas de alto factor de impacto se ha traducido en un aumento de las prácticas de "autoría honorífica", en un intento por maximizar la productividad en términos de publicaciones ${ }^{16}$.

Es de interés un estudio que encuestó a 328 primeros autores de publicaciones en el área de la radiología ${ }^{18}$. En éste, el 27,7\% de los primeros autores percibió que al menos un coautor no realizó lo suficiente para merecer la autoría en el estudio y el 50,3\% afirmó que uno o más coautores sólo habían realizado tareas no propias de "autorías"18. En un trabajo posterior de los mismos autores, se revela que la percepción de "autorías honoríficas" fue significativamente mayor en los primeros autores de instituciones asiáticas y europeas, comparado con los de instituciones norteamericanas. La mayor autoría honorífica se aprecia también en instituciones donde el jefe de departamento era listado de manera automática como coautor ${ }^{19}$. También se otorgan autorías inmerecidas como una forma de retribución recíproca ${ }^{13}$. Cuando se utiliza apropiadamente, la autoría ofrece un historial de rendición de cuentas, de responsabilidad y el correspondiente crédito por el trabajo realizado; sin embargo, un número creciente de académicos de alto nivel dan más valor al número de sus publicaciones en lugar de su contribución al conocimiento ${ }^{3}$.

\section{Autores fantasmas y autores invitados}

Por autores fantasmas se entiende a aquel autor que habiendo realizado contribución fundamental en un manuscrito, que lo calificaría para aparecer en la lista de autores, no es mencionado en ella ${ }^{20}$. $\mathrm{Su}$ existencia ha aumentado en publicaciones biomédicas, estimándose que aproximadamente un $10 \%$ de los artículos tiene autores fantasmas ${ }^{20}$. El principal problema asociado a esta práctica es que esconden evidentes conflictos de intereses, puesto que suelen ser financiados por la industria farmacéutica o de servicios médicos para promocionar sus productos. Hay que hacer presente que la industria no sólo financia ensayos clínicos, sino que también patrocina revisiones sistemáticas, meta-análisis, estudios de costo efectividad, entre otros, que pueden influir en la prescripción ${ }^{10}$.

Otra práctica se refiere al pago a científicos o académicos que prestan su nombre -como autores invitados-, para aparecer avalando revisiones a favor de un determinado producto, sin haber contribuido intelectualmente en la publicación ${ }^{10}$. Con prácticas de esta naturaleza, las compañías logran promocionar sus productos desde una posición que aparece como académica e independiente, mediante el uso de autores invitados que prestan su nombre para estas prácticas poco éticas, vulnerando así los valores básicos de la ciencia, la validez del conocimiento científico y, en consecuencia, el mismo cuidado del paciente está en riesgo ${ }^{20}$. Un ejemplo fue cómo autores invitados, financiados por una compañía farmacéutica, escribieron sobre las consecuencias favorables del uso de terapia de reemplazo hormonal, minimizando los eventos adversos, lo que impactó en la salud de miles de mujeres ${ }^{20,21}$. Es relevante diferenciar esta práctica no ética, del uso correcto de escritores profesionales, que permiten mejorar la escritura, ajustar el manuscrito a los requerimientos de una determinada revista 
y disminuir las eventuales barreras idiomáticas; incluso más, como lo muestran trabajos recientes, su participación contribuiría a una mejoría del reporte de los ensayos clínicos ${ }^{22}$.

\section{Irregularidades en la publicación de resultados}

La autoría provee reconocimiento entre los pares y establece credibilidad profesional e intelectual, contribuyendo a la progresión académica de los investigadores. Estos beneficios, sin embargo, conllevan responsabilidades para difundir los resultados de la investigación ${ }^{23}$. Es crucial que las personas asignadas como autores en las investigaciones hayan realmente realizado el trabajo y estén calificadas para garantizar los resultados ${ }^{24}$. Sin embargo, el trabajo en equipo propicia conflictos que pueden interferir o impedir la publicación de la investigación de manera adecuada y en los tiempos estipulados. Las irregularidades más frecuentes en la publicación de resultados pueden dividirse en las siguientes categorías:

\section{Malas prácticas científicas}

Tales como el plagio, la fabricación o falsificación de resultados, el infringir el protocolo de investigación, la coerción de los sujetos de estudio, y la omisión de resultados, entre otras ${ }^{25}$. Una investigación realizada en Nigeria demostró que el $68,9 \%$ de los autores admitió haber realizado al menos una forma de mala práctica, siendo las más frecuentes el desacuerdo entre autorías y la falsificación de datos o plagio ${ }^{26}$. Este tipo de malas prácticas puede tener diversas consecuencias, dependiendo de la naturaleza del problema y del curso que tome la resolución del conflicto, que enlentecen los tiempos de publicación o pueden llevar a la no publicación de los resultados o causar la divulgación de resultados incorrectos o plagiados ${ }^{25,26}$.

\section{Conflictos de autoría}

Los conflictos de autoría pertenecen a las malas prácticas científicas, pero merecen mención aparte por poseer una alta frecuencia ${ }^{26}$. Los ensayos clínicos atraviesan barreras institucionales e internacionales, que requieren establecer relaciones entre colaboradores; esta interdependencia podría eventualmente llevar a conflictos de autoría ${ }^{24}$. Dentro de los conflictos de autoría que han ido en aumento en los últimos años, podemos men- cionar la coautoría no correspondiente, originada entre investigadores que compiten por el puesto de investigador principal ${ }^{25}$. Otros conflictos de autoría afectan a los investigadores con participación parcial en el proyecto o investigadores con contratos a corto plazo, que muchas veces por traslados o términos de contrato pierden la autoría correspondiente en las investigaciones donde participaron parcialmente $e^{24,25}$.

\section{Conflictos de propiedad de artículos científicos} entre investigadores y compañias patrocinadoras

Muchas veces se producen desacuerdos entre estas entidades en cuanto a los derechos de publicación de resultados y su aprobación. Cuando los investigadores ceden estos derechos a las compañías patrocinadoras, si los resultados no van en su beneficio, éstas pueden obstaculizar la publicación. Se recomienda evitar que los investigadores entreguen el poder de publicación a las compañías y de esta forma mantener la libertad académica, para lo cual es necesario revisar previamente el contrato ${ }^{25}$.

\section{Criterios de autoría en trabajos colaborativos internacionales}

En los últimos años, se ha desarrollado el concepto de "salud global", que tiene como prioridad la mejoría y el logro de equidad en salud para todas las personas, involucrando a muchas disciplinas que requieren colaboración interdisciplinaria internacional, propiciando la publicación de artículos de multiautoría ${ }^{27,28}$.

Según los criterios de ICMJE, en estos casos el grupo debe idealmente decidir quién será el autor principal antes del inicio del trabajo y confirmar los autores previo al envío del manuscrito a publicar $^{4}$. ICMJE establece los criterios en caso de multiautoría, pero no especifica criterios en el contexto de equipos internacionales multidisciplinarios ${ }^{4}$. Muchas veces los trabajos multidisciplinarios implican alianzas de salud global, que requiere una distribución equitativa de las autorías entre países de bajo y medio ingreso con respecto a países de altos ingresos, lo que provoca conflictos, agravados por las diferencias en los niveles de inglés entre los investigadores de diferentes países, que dificulta aún más el cumplimiento de todos los requisitos de autoría del ICMJE $\mathrm{E}^{4,28,29}$. 
Otro conflicto frecuente obedece al orden de autoría en las publicaciones; generalmente éste se establece de forma descendiente de contribución, pero puede ser de acuerdo a otros criterios como orden alfabético, o investigador principal como último autor ${ }^{14}$. Las normas a este respecto no han sido formalmente establecidas y eso puede llevar a reconocimiento insuficiente de ciertos autores, produciendo conflictos. Por último, existen conflictos de autoría respecto a sesgos editoriales que favorecen con la primera autoría a investigadores de países de altos ingresos, por creer que tendrán mayores oportunidades de publicación en revistas de alto impacto con respecto a investigadores de países de ingresos bajo-medios; también existen conflictos de autoría según contexto cultural que involucran sesgos de género o autoría asignada a personas con extensa trayectoria académica ${ }^{28}$. Para evitar este tipo de conflictos, se ha propuesto nombrar a los coautores potenciales de cada centro e incluso firmar contratos legales de autoría previo al inicio del estudio ${ }^{30}$.

\section{Recomendaciones y Conclusión}

El ICMJE señala que la autoría de las publicaciones confiere reconocimiento y tiene importantes consecuencias académicas, sociales y financieras para los autores; asimismo, una correcta asignación de autoría a las personas debidamente implicadas las lleva a rendir cuentas por los contenidos del trabajo publicado, asumiendo responsabilidad pública del mismo ${ }^{4}$. No obstante existir diversas normas y guías referentes a la correcta asignación de autorías, los conflictos persisten. La integridad de las publicaciones depende del compromiso ético de cada autor con la veracidad y honestidad intelectual de lo que se reporta, lo que en el área biomédica puede tener importante impacto en la salud de las personas. Asimismo, una buena ética de la publicación desaprueba tanto al autor honorario como al autor fantasma ${ }^{15}$.

Dado lo controversial del tema, recomendamos que la asignación de autoría sea definida antes de comenzar el trabajo. Es importante que exista adecuado conocimiento de las guías y recomendaciones que efectúan las diversas entidades que se han pronunciado sobre el tema, para lo cual se debieran incluir estos aspectos en la docencia de pre y postgrado. Finalmente sugerimos que las instituciones académicas, responsables de haber influido en convertir la métrica de las publicaciones en el más alto estándar de la labor académica, orienten y guíen respecto del tema o modifiquen el sistema actual, tal como ha sido mencionado por algunos autores ${ }^{31}$.

\section{Referencias}

1. Wager E, Kleinert S. Responsible research publication: international standards for authors. A position statement developed at the 2 nd World Conference on Research Integrity, Singapore, July 22-24, 2010. 2011; Chapter 50 in: Mayer T \& Steneck N (eds) Promoting Research Integrity in a Global Environment. Imperial College Press / World Scientific Publishing, Singapore (pp 309-16). (ISBN 978-981-4340-97-7).

2. Biagioli M y Galison P (eds). Scientific Authorship: Credit and Intellectual Property in Science. New York, Routledge, 2003. ISBN 0415942926.

3. Wagena EJ. The scandal of unfair behaviour of senior faculty. J Med Ethics 2005; 31 (5): 308.

4. International Committee of Medical Journal Editors. Recommendations for the conduct, reporting, editing, and publication of scholarly work in medical journals. Updated December 2015. Disponible en http://www. icmje.org/icmje-recommendations.pdf. Fecha de acceso 7 de julio de 2016.

5. International Committee of Medical Journal Editors. Journals Following the ICMJE Recommendations. Disponible en http://www.icmje.org/journals-following-the-icmje-recommendations/ - R. Fecha de acceso 10 de marzo de 2016.

6. Revista Médica de Chile. Instrucciones a los autores. Disponible en http://www.scielo.cl/revistas/rmc/einstruc.htm. Fecha de acceso 23 de diciembre de 2015.

7. Revista Chilena de Pediatría. Instrucciones a los autores. Disponible en http://www.scielo.cl/revistas/rcp/einstruc. htm. Fecha de acceso 4 enero de 2016.

8. Reyes HB. La relevancia del International Committee of Medical Journal Editors (ICMJE) para las publicaciones y la investigación médica. Rev Med Chile 2014; 142 (1): 79-83.

9. Ley 17.336. Propiedad Intelectual, Derecho de autor. Promulgada el 28 de agosto de 1970 por el Ministerio de Educación Pública de Chile. Disponible en http:// bcn.cl/1m035. Fecha de acceso 10 de enero de 2016.

10. Sismondo S, Doucet M. Publication ethics and the ghost management of medical publication. Bioethics 2010; 24 (6): 273-83. 
11. Surgical Journal Editors Group. Consensus statement on surgery journal authorship-2006. Ann Surg 2006; 243 (6): 713-4.

12. Aguzzi A. Authoring scientific papers: a perspective from the trenches. Swiss Med Wkly 2015; 145: w14107.

13. Albert T, Wager E. How to handle authorship disputes: a guide for new researchers. Reporte anual del Committee on publication ethics (COPE). Disponible en http://publicationethics.org/annualreport/2003. Fecha de acceso 21 de marzo de 2016. 2003.

14. Scientific Integrity Committee of Swiss Academies of Arts Sciences, Hess CW, Bruckner C, Kaiser T, Mauron A, Wahli W, et al. Authorship in scientific publications: analysis and recommendations. Swiss Med Wkly 2015; 145: w14108.

15. Avula J, Avula H. Authors, authorship order, the moving finger writes. J Indian Soc Periodontol 2015; 19 (3): 25862.

16. Walker RL, Sykes L, Hemmelgarn BR, Quan H. Authors' opinions on publication in relation to annual performance assessment. BMC Med Educ 2010; 10: 21.

17. Marusic A, Bosnjak L, Jeroncic A. A systematic review of research on the meaning, ethics and practices of authorship across scholarly disciplines. PLoS One 2011; 6 (9): e23477.

18. Eisenberg RL, Ngo L, Boiselle PM, Bankier AA. Honorary authorship in radiologic research articles: assessment of frequency and associated factors. Radiology 2011; 259 (2): 479-86.

19. Eisenberg RL, Ngo LH, Bankier AA. Honorary authorship in radiologic research articles: do geographic factors influence the frequency? Radiology 2014; 271 (2): 472-8.

20. Gorski A, Letkiewicz S. "Medical writing" and ghostwriting as ethical challenges in medical communication. Transplant Proc 2010; 42 (8): 3335-7.

21. Fugh-Berman AJ. The haunting of medical journals: how ghostwriting sold "HRT". PLoS Med 2010; 7 (9): e1000335.
22. Gattrell WT, Hopewell S, Young K, Farrow P, White R, Wager E, et al. Professional medical writing support and the quality of randomised controlled trial reporting: a cross-sectional study. BMJ Open 2016; 6 (2): e010329.

23. Lundberg GD, Glass RM. What does authorship mean in a peer-reviewed medical journal? JAMA 1996; 276 (1): 75.

24. Newman A, Jones R. Authorship of research papers: ethical and professional issues for short-term researchers. J Med Ethics 2006; 32 (7): 420-3.

25. Responsible Authorship And Peer Review. Responsible conduct of research, Universidad de Columbia. Disponible en http://ccnmtl.columbia.edu/projects/ rcr/rcr_authorship/introduction/index.html. Fecha de acceso 10 de enero de 2016.

26. Okonta P, Rossouw T. Prevalence of scientific misconduct among a group of researchers in Nigeria. Dev World Bioeth 2013; 13 (3): 149-57.

27. Koplan JP, Bond TC, Merson MH, Reddy KS, Rodriguez $\mathrm{MH}$, Sewankambo NK, et al. Towards a common definition of global health. Lancet 2009; 373 (9679): 1993-5.

28. Smith E, Hunt M, Master Z. Authorship ethics in global health research partnerships between researchers from low or middle income countries and high income countries. BMC Med Ethics 2014; 15: 42.

29. Zachariah R, Reid T, Van den Bergh R, Dahmane A, Kosgei RJ, Hinderaker SG, et al. Applying the ICMJE authorship criteria to operational research in low-income countries: the need to engage programme managers and policy makers. Trop Med Int Health 2013; 18 (8): 1025-8.

30. Rosenberg J, Burcharth J, Pommergaard HC, Vinther S. Authorship issues in multi-centre clinical trials: the importance of making an authorship contract. Dan Med J 2015; 62 (2).

31. Bennett DM, Taylor DM. Unethical practices in authorship of scientific papers. Emerg Med (Fremantle) 2003; 15 (3): 263-70. 